\title{
WS13 A01
}

Mimetic Operators for Seismic Exploration

\section{J. de la Puente* (Barcelona Supercomputing Center), O. Rojas (Barcelona Supercomputing Center), M. Ferrer (Barcelona Supercomputing Center) \& J. Kormann (Barcelona Supercomputing Center)}

\section{SUMMARY}

Mimetic operators are a kind of discrete operators which, on staggered grids, can accomplish "mimetic" properties analogous to their continuous counterparts. Furthermore, they can attain identical convergence order everywhere in the domain, including at or near the domain's boundaries in non-periodic problems. This property makes mimetic finite difference (MFD) operators attractive whenever high-contrast interfaces or physical boundary conditions are present in our models. In the seismic case, the strongest boundary condition is the interface between Earth and air, the so-called free surface. This surface is typically represented as a traction-free boundary condition and, although it is of critical importance for achieving accurate results, due to its vicinity to the sources and receivers, it is still a problem for current FD implementations. Despite efforts in the past, few FD schemes have attained convergence of order beyond two in results involving the free surface. Here we summarize the properties of mimetic operators applied to the elastic wave equation, as well as the changes required in order to incorporate into current explicit staggered grid codes the mimetic approach of the free-surface condition 


\section{Introduction}

Whenever finite difference (FD) methods are evaluated in terms of their efficiency and accuracy, it is customary to use periodic or harmonic problems where the properties of the method are actually guaranteed for infinite (and smooth) media. However, it is widely known, that FD operators do not preserve their properties at domain boundaries, especially under high-order discretizations. Typical one-sided operators can be built based upon Taylor's expansion with an arbitrary order of accuracy (Fornberg, 1988). Nevertheless, these operators become inaccurate and some of the properties of FD in the infinite space may no longer be valid. Mimetic operators are a kind of discrete operators which, on staggered grids, can accomplish mimetic properties analogous to their continuous counterparts (Castillo et al., 2001; Castillo and Miranda, 2013). Furthermore, they can attain identical convergence order everywhere in the domain, including at or near the domain's boundaries in non-periodic problems. This property makes mimetic finite difference (MFD) operators attractive whenever high-contrast interfaces or physical boundary conditions are present in our models. In the seismic case, the strongest boundary condition is the interface between Earth and air, the so-called free surface. This surface is typically represented as a traction-free boundary condition and, although it is of critical importance for achieving accurate results, due to its vicinity to the sources and receivers, it is still a problem for current FD implementations. Despite efforts in the past, few FD schemes have attained convergence (e.g. Kristek et al., 2002) of order beyond two in results involving the free surface. Here, we apply mimetic operators to model elastic motion under free-surface conditions and discuss the implementation changes required by current explicit staggered grid codes to include this mimetic approach.

\section{Theory}

The elastodynamic equations can be written as

$$
\begin{aligned}
& \rho(\mathbf{x}) \dot{\mathbf{v}}(\mathbf{x}, t)=\nabla \cdot \sigma(\mathbf{x}, t)+\mathbf{f}_{s}\left(\mathbf{x}_{s}, t\right), \\
& \dot{\boldsymbol{\sigma}}(\mathbf{x}, t)=\mathbf{C}(\mathbf{x}): \nabla \mathbf{v}(\mathbf{x}, t)
\end{aligned}
$$

where $f_{\mathrm{s}}$ is the source function at position $\mathbf{x}_{\mathrm{s}}, \mathbf{v}$ is the particle velocity, $\rho$ is the density, and $\sigma$ the stress. At the free surface, $z=0$ for convenience, a Dirichlet boundary condition $C$ must be fulfilled, namely $\sigma \cdot \mathbf{n}=0$ with $\mathbf{n}$ a unit vector perpendicular to the free surface. We can reduce the condition in the flat case to

$$
\begin{aligned}
& C 1: \sigma_{x z}=0, \sigma_{y z}=0, \\
& C 2: \sigma_{z z}=0 .
\end{aligned}
$$

Figure 1 shows the modifications to standard staggered grids (SSG, e.g. Virieux, 1986; Rojas et al., 2008) and fully staggered grids (FSG, e.g. Lebedev, 1964; de la Puente et al., 2014) resulting from updating their original Leap-Frog 2 explicit time-integration schemes to accomodate the mimetic approach. We use a two-dimensional representation for simplicity. Notice that interior nodes are updated as usual without further modifications. In the figure, variables in black are natural variables of that grid type, grey variables are natural but become zero due to the boundary condition $C$ and green variables are additional variables that appear only when using mimetic schemes and only at the free surface. The latter are labelled with an $M$ superindex depicting their mimetic origin. Both classes of SSG freesurface possibilities are shown, as well as the flat and tilted FSG cases. Notice that in the non-flat case the free surface is defined at the curved surface $\eta=0$. In addition, the boundary condition $C$ cannot be simplified to 2 and requires special treatment (details shown by de la Puente et al., 2014).

The vertical derivatives $d$ are solved by means of mimetic differentiation operators $G$ and $D$ (gradient 


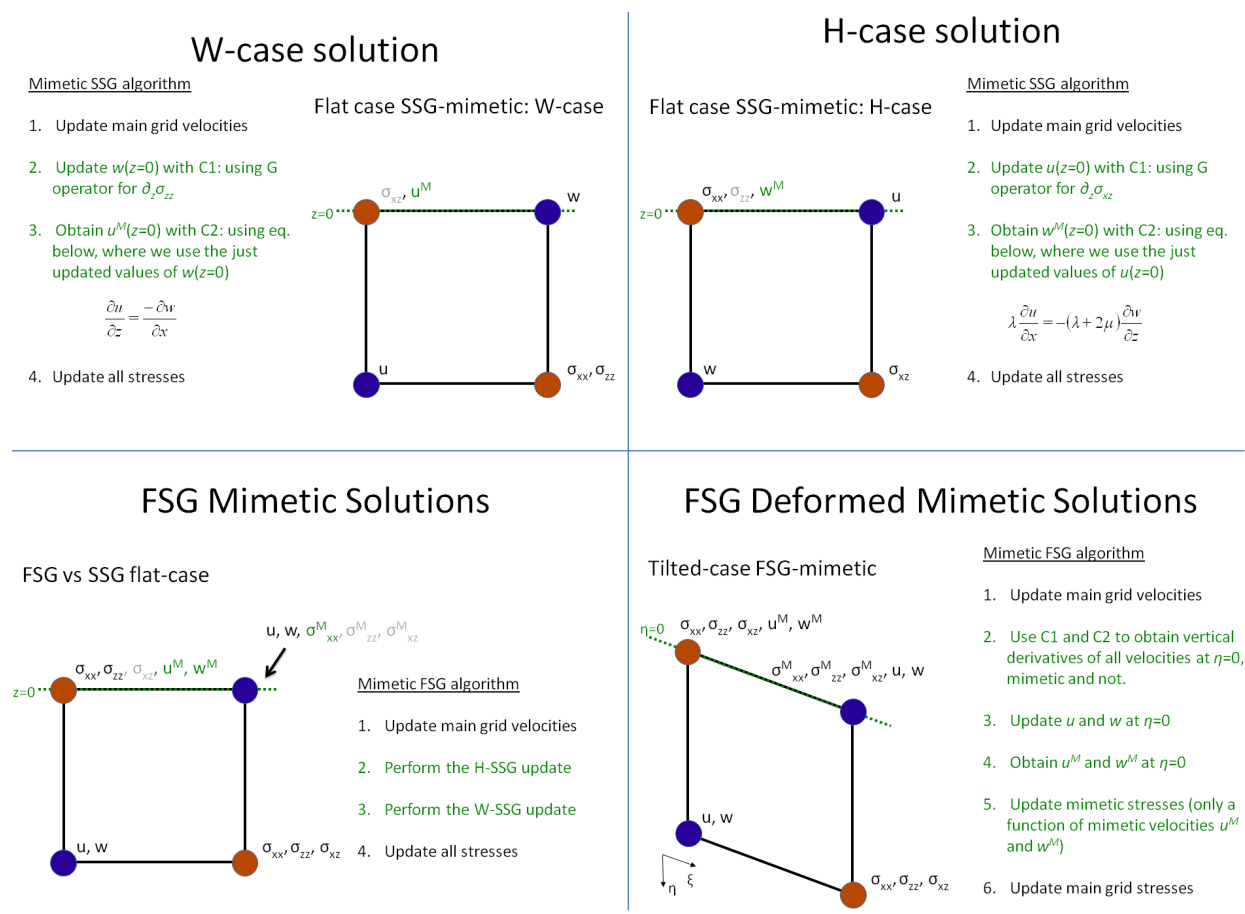

Figure 1 Algorithmics for mimetic methods applied to the free-surface in SSG of types $W$ and $H$ (top) and FSG flat and deformed (bottom). We use $u$ and $w$ to depict horizontal and vertical components of $\mathbf{v}$ here.

and divergence) following

$$
\begin{gathered}
d_{K}^{B} a(I, J, K)=\frac{1}{\Delta} \sum_{r=1}^{n^{D}+1} D_{K r} a(I, J, K+r-1), \\
d_{K}^{F} a(I, J, K)=\frac{1}{\Delta} \sum_{r=2}^{n^{G}+1} G_{K r} a(I, J, K+r-1)+G_{11} a^{M}(I, J) .
\end{gathered}
$$

where $a$ is the differentiated variable, $(I J K)$ the cell index in $x-, y$ - and $z$-directions ( $K=1$ corresponding to $z=0$ or $\eta=0$ ), $\Delta$ the vertical spacing, $G_{K r}$ and $D_{K r}$ are the $r$ th coefficient of the $K t h$ specialized stencil of the corresponding mimetic operator and $n^{G}, n^{D}$ their respective bandwidth. Finally, whenever $a$ is a natural variable at the free-surface (e.g. $w$ in the $\mathrm{W}$-case of Figure 1) the first operator in 3 is used (labelled B) and otherwise the latest operator is used (labelled F). The actual stencil values can be found in, e.g. Rojas et al. (2009) or Castillo and Miranda (2013).

\section{Example}

We show a simple flat case with free surface, involving three layers. We run the case both with the vacuum formulation (Robertsson, 1996; Zeng et al., 2012) and with the mimetic approach described here. In Figure 3 we can see that the snapshots are rather similar, as both approaches describe approximations to the same physical problem. Nevertheless, an inspection of vertical component traces shows us how, the farther surface waves travel, the more dispersion and phase shift appears in the vaccuum case, related to its lack of formal accuracy (order 1). 

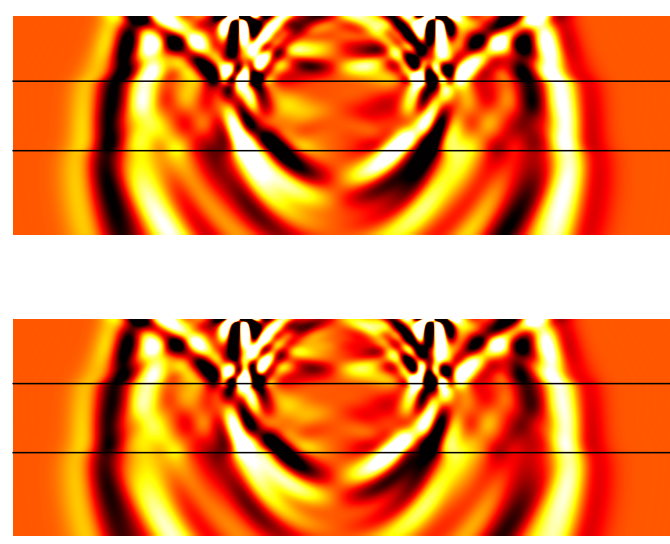

(a)

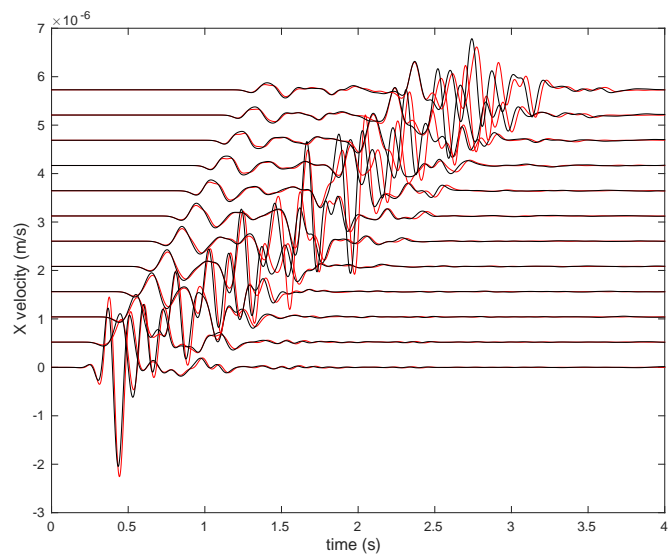

(b)

Figure 2 (a) Horizontal component snapshots obtained with the mimetic approach (top) and vacuum approach (bottom) for the same scenario. (b) Traces obtained with mimetic (black) and vacuum (red) algorithms.
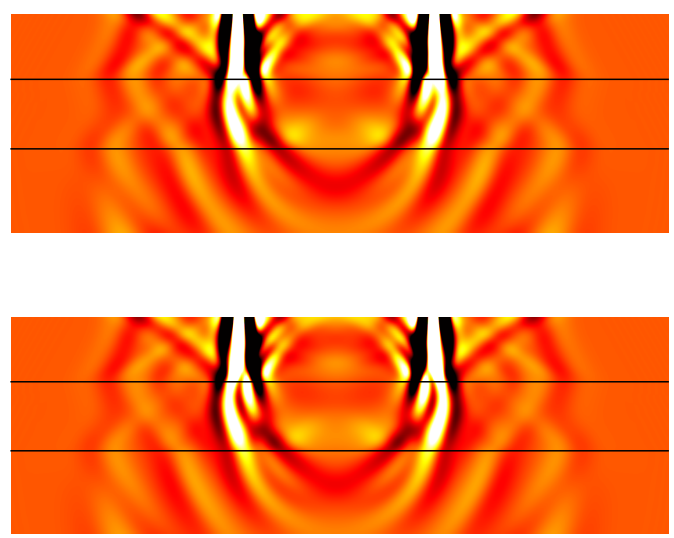

(a)

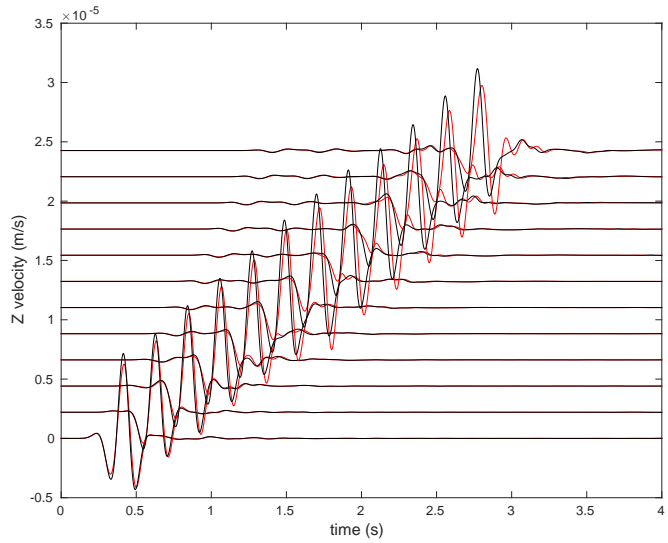

(b)

Figure 3 (a) Vertical component snapshots obtained with the mimetic approach (top) and vacuum approach (bottom) for the same scenario. (b) Traces obtained with mimetic (black) and vacuum (red) algorithms.

\section{Conclusions}

The mimetic approach is a simple upgrade for existing staggered-grid codes that can yield important gains accuracy-wise in the presence of large material contrasts, such as the free surface. The simplicity of the approach has allowed us to develop 3D imaging tools that make use of its enhanced accuracy. During our talk we will also show examples of its application in migration and inversion scenarios.

\section{Acknowledgements}

This project has received funding from the European Union's Horizon 2020 research and innovation programme under the Marie Sklodowska-Curie grant agreement No 644602. The research leading to these results has received funding from the European Union's Horizon 2020 Programme (2014-2020) 
and from Brazilian Ministry of Science, Technology and Innovation through Rede Nacional de Pesquisa (RNP) under the HPC4E Project (www.hpc4e.eu), grant agreement No 689772.

\section{References}

Castillo, J., Hyman, J., Shashkov, M. and Steinberg, S. [2001] Fourth- and sixth-order conservative finite difference approximations of the divergence and gradient. Appl. Numer. Math., 37(1-2), 171-187.

Castillo, J.E. and Miranda, G.F. [2013] Mimetic Discretization Methods. CRC Press, Boca Raton, Florida.

Fornberg, B. [1988] Generation of Finite Difference Formulas on Arbitrary Spaced Grids. Mathematics of Computation, 51(184), 699-706.

Kristek, J., Moczo, P. and Archuleta, R.J. [2002] Efficient Methods to Simulate Planar Free Surface in the 3D 4th-Order Staggered-Grid Finite-Difference Schemes. Studia Geophysica et Geodaetica, 46, 355-381. 10.1023/A:1019866422821.

Lebedev, V. [1964] Difference analogies of orthogonal decompositions of basic differential operators and some boundary value problems. I. Sov. Comput. Math. Math. Phys., 4, 449-465.

de la Puente, J., Ferrer, M., Hanzich, M., Castillo, J.E. and Cela, J.M. [2014] Mimetic seismic wave modeling including topography on deformed staggered grids. Geophysics, 79(3), T125-T141.

Robertsson, J.O.A. [1996] A numerical free-surface condition for elastic/ viscoelastic finite-difference modeling in the presence of topography. Geophysics, 61(6), 1921-1934.

Rojas, O., Day, S., Castillo, J. and Dalguer, L.A. [2008] Modelling of rupture propagation using highorder mimetic finite differences. Geophys. J. Int., 172(2), 631-650.

Rojas, O., Dunham, E.M., Day, S.M., Dalguer, L.A. and Castillo, J.E. [2009] Finite difference modelling of rupture propagation with strong velocity -weakening friction. Geophysical Journal International, 179(3), 1831-1858.

Virieux, J. [1986] P-SV wave propagation in heterogeneous media: Velocity-stress finite-difference method. Geophysics, 51, 889-901.

Zeng, C., Xia, J., Miller, R.D. and Tsoflias, G.P. [2012] An improved vacuum formulation for 2D finitedifference modeling of Rayleigh waves including surface topography and internal discontinuities. Geophysics, 77(1), T1-T9. 